\title{
Observer-Type Consensus Protocol for a Class of Fractional-Order Uncertain Multiagent Systems
}

\author{
Hongjie Li \\ College of Mathematics, Physics and Information Engineering, Jiaxing University, Zhejiang 314001, China \\ Correspondence should be addressed to Hongjie Li, lhjlyyml@163.com
}

Received 7 May 2012; Revised 9 July 2012; Accepted 3 August 2012

Academic Editor: Bashir Ahmad

Copyright (C) 2012 Hongjie Li. This is an open access article distributed under the Creative Commons Attribution License, which permits unrestricted use, distribution, and reproduction in any medium, provided the original work is properly cited.

This paper investigates the consensus problem for a class of fractional-order uncertain multiagent systems with general linear node dynamics. Firstly, an observer-type consensus protocol is proposed based on the relative observer states of neighboring agents. Secondly, based on property of the Kronecker product and stability theory of fractional-order system, some sufficient conditions are presented for robust asymptotical stability of the observer-based fractional-order control systems. Thirdly, robust stabilizing controllers are derived by using linear matrix inequality approach and matrix's singular value decomposition. Our results are in the form of linear matrix inequalities which can easily be solved by LMI toolbox in MATLAB. Finally, a numerical simulation is performed to show the effectiveness of the theoretical results.

\section{Introduction}

The coordination problem of multiagent dynamical systems have attracted an increasing attention in recent years due to its applications in sensor networks, robotic teams, satellites formation [1-3]. Particularly, the consensus of multiagent systems, which has been extensively studied in the past few years [4-8].

Note that for most of aforementioned results, the agent dynamics is assumed to be first-order, second-order, or high-order integrators, which may be restrictive in many cases. Recently, consensus of multiagent systems with general linear node dynamics has received dramatic attention [9-11], the output feedback consensus problem is studies in [9] for high-order linear system, the consensus is reached if there exists a stable compensator. In [10], the consensus problem of multiagent systems with general form of linear dynamics is investigated under a time-invariant communication topology, an observer-type consensus protocol based on relative output measurements between neighboring agents has been 
proposed. Li et al. [11] investigate the consensus problems for multiagent systems with continuous-time and discrete-time linear dynamics, distributed reduce-order consensus protocols have been proposed based on the information of relative outputs of neighboring agents. In the above-mentioned works on consensus of multiagent systems, the systems are described by an integer-order dynamics, but many phenomena in nature cannot be explained in the framework of integer-order dynamics, for example, the synchronized motion of agents in fractional circumstances, such as molecule fluids and porous media, the stress-strain relationship demonstrates non-integer-order dynamics rather than integer-order dynamics [12]. In addition, fractional-order systems provide an excellent instrument for the description of memory and hereditary properties of various materials and processes, such as dielectric polarization, electrode-electrolyte polarization, and visco-elastic systems [13-16]. For the fractional-order dynamical systems, it is very difficult and inconvenient to construct Lyapunov functions, because there exist substantial differences between fractional-order differential systems and integer-order differential ones. As a way of efficiently solving the robust stability and stabilization problem, the linear matrix inequality approach is presented [17-20], which provides the designing method of state feedback controllers for fractional-order systems. A recent study [21] investigates the distributed formation control problem for multiple fractional-order systems under dynamic interaction, sufficient conditions on the network topology are given to ensure the formation control. In [21], the coordination algorithms for networked fractional-order systems are studied when the fixed interaction graph is directed, the coordination algorithms for integer-order system can be considered a special case of those for fractional-order systems. Only two of the above studies considered fractional-order multiagent systems. In addition, in previous results, static consensus protocols based on relative states of neighboring agents are used, which require the absolute output measurement of each agent to be available, which is impractical in many cases, the agent states in relatively large scale networks are not often completely available. Thus, in many application, one often needs to estimate the agent states through available measurements and then utilizes the estimated states to achieve certain design objectives such as consensus of multiagent systems, synchronization of complex networks [22-25]. To date, very little research effort has been done about the consensus problem for fractional-order uncertain multiagent systems. The purpose of our study is to fill this gap.

Motivated by the above discussion, the consensus problem is investigated for a class of fractional-order uncertain multiagent systems with general linear node dynamics. An observer-type consensus protocol is proposed based on the relative observer states of neighboring agents. Some sufficient conditions are presented for robust asymptotical stability of the observer-based fractional-order control systems, and robust stabilizing controllers are derived by using linear matrix inequality approach and matrix's singular value decomposition. Finally, an illustrative example is provided to demonstrate the effectiveness of the proposed approach.

The main novelties of this study are summarized as follows: (1) a novel observertype consensus protocol is proposed based on the relative observer states of neighboring agents. Different from [26], the observer state is used instead of the agent's state in consensus protocol, and the dynamic behavior of multiagent is described by fractional-order system; (2) the uncertainty is considered in multiagent systems due to external disturbing factors such as environment temperature, voltage fluctuation, and mutual interfere among components; (3) the feedback gain matrices can be derived by matrix's singular value decomposition, and the consensus criteria are in the form of linear matrix inequalities which can be solved by applying the LMI toolbox. 
The rest of this paper is organized as follows. In Section 2, preliminaries and problem statement are given. In Section 3, the consensus conditions are derived by using linear matrix inequality approach and matrix's singular value decomposition. In Section 4, a simulation example is provided to show the advantages of the obtained results. Conclusions are presented in Section 5.

\section{Preliminaries and Problem Statement}

\subsection{Graph Theory Notions}

Let $g=\{v, \varepsilon, A\}$ be a weighted directed graph of order $N$, with the set of nodes $v=$ $\left\{v_{1}, v_{2}, \ldots, v_{N}\right\}$, an edge set $\varepsilon \subseteq v \times v$, and a weighted adjacency matrix $A=\left(a_{i j}\right)_{N \times N}$ with $a_{i j}>0$ if $\left(v_{j}, v_{i}\right) \in \varepsilon$ and $a_{i j}=0$, otherwise. The neighbor set of node $i$ is defined by $N_{i}=\left\{j \in v \mid\left(v_{j}, v_{i}\right) \in \varepsilon\right\}$, and the in-degree and out-degree of node $i$ are defined as

$$
\operatorname{deg}_{\text {in }}(i)=\sum_{j=1, j \neq i}^{N} a_{i j}, \quad \operatorname{deg}_{\text {out }}(i)=\sum_{j=1, j \neq i}^{N} a_{j i} .
$$

A diagraph is called balanced if $\operatorname{deg}_{\text {in }}(i)=\operatorname{deg}_{\text {out }}(i)$ for all $i \in v$.

The Laplacian matrix $L=\left(l_{i j}\right)_{N \times N}$ associated with the adjacency matrix $A$ is defined as

$$
\begin{gathered}
l_{i j}=-a_{i j} \quad(i \neq j), \\
l_{i i}=-\sum_{j=1, j \neq i}^{N} a_{i j}, \quad(i=1,2, \ldots, N) .
\end{gathered}
$$

It is straightforward to verify that $L$ has at least one zero eigenvalue with a corresponding eigenvalue with a corresponding eigenvector $\mathbf{1}$, where $\mathbf{1}$ is an all-one column vector with a compatible size.

\subsection{Caputo Fractional Operator}

With the development of fractional calculus, it has been found that many physical systems show fractional dynamical behavior because of special materials and chemical properties, which can be described more accurately using fractional-order calculus than traditional integer-order calculus $[27,28]$. Therefore, fractional-order calculus has become a hot research issue in recent years. There are many definitions of fractional derivatives [29-31], such as the Riemann-Liouville derivative and the Caputo derivative which are used in fractional systems. In physical systems, Caputo fractional derivative is more appropriate for describing the initial value problem of fractional differential equations, the Laplace transform of the Caputo derivative allows utilization of initial values of classical integer-order derivatives 
with clear physical interpretations [17]. Therefore, the following Caputo fractional operator is adopted in this paper for fractional derivatives of order $\alpha$ :

$$
D^{\alpha} x(t)=\frac{1}{\Gamma(m-\alpha)} \int_{t_{0}}^{t}(t-\tau)^{m-\alpha-1} x^{(m)}(\tau) d \tau \quad(m-1<\alpha<m)
$$

where $m \in \mathbb{Z}_{+}, \Gamma(\cdot)$ is a gamma function given by $\Gamma(z)=\int_{0}^{\infty} t^{z-1} e^{-t} d t$.

In order to simulate the fractional-order multiagent systems, a predictor corrector algorithm is introduced as follows.

The fractional-order differential equation is given by

$$
\begin{gathered}
D^{\alpha} x(t)=f(t, x(t)) \quad(0 \leq t \leq T, 0<\alpha<1), \\
x^{(i)}(0)=x_{0}^{(i)} \quad(i=0,1,2, \ldots, n-1)
\end{gathered}
$$

which is equivalent to the following Volterra integral equation:

$$
x(t)=\sum_{i=0}^{\lceil a\rceil-1} \frac{t^{i}}{i !} x_{0}^{(i)}+\frac{1}{\Gamma(\alpha)} \int_{0}^{t}(t-\tau)^{\alpha-1} f(\tau, x(\tau)) d \tau .
$$

Set $h=T / N \quad\left(N \in \mathbb{Z}_{+}\right)$and $t_{n}=n h(n=1,2, \ldots, N)$, where $h$ is the step size, $T$ is simulation time, and $N$ is the number of sample points, (2.4) can be discretized as follows:

$$
\begin{aligned}
x_{h}\left(t_{n+1}\right)= & \sum_{i=0}^{\lceil a\rceil-1} \frac{t_{n+1}^{i}}{i !} x_{0}^{(i)}+\frac{h^{\alpha}}{\Gamma(\alpha+2)} f\left(t_{n+1}, x_{h}^{p}\left(t_{n+1}\right)\right) \\
& +\frac{h^{\alpha}}{\Gamma(\alpha+2)} \sum_{j=0}^{n} a_{j, n+1} f\left(t_{j}, x_{h}\left(t_{j}\right)\right),
\end{aligned}
$$

where

$$
\begin{aligned}
x_{h}^{p}\left(t_{n+1}\right) & =\sum_{i=0}^{n-1} \frac{t_{n+1}^{i}}{i !} x_{0}^{(i)}+\frac{1}{\Gamma(\alpha)} \sum_{j=0}^{n} b_{j, n+1} f\left(t_{j}, x_{h}\left(t_{j}\right)\right), \\
a_{j, n+1} & = \begin{cases}n^{\alpha+1}-(n-\alpha)(n+1)^{\alpha+1} & (j=0) \\
(n-j+2)^{\alpha+1}+(n-j)^{\alpha+1}-2(n-j+1)^{\alpha+1} & (1 \leq j \leq n) \\
1 & (j=n+1),\end{cases} \\
b_{j, n+1}= & \frac{h^{\alpha}}{\alpha}\left((n-j+1)^{\alpha}-(n-j)^{\alpha}\right),
\end{aligned}
$$

the estimation error of this approximation is $e=\max _{j=0,1, \ldots, N}\left|x\left(t_{j}\right)-x_{h}\left(t_{j}\right)\right|=O\left(h^{p}\right)$, where $p=\min (2,1+\alpha)$. 


\subsection{Problem Formulation}

Consider multiagent systems of $N$ identical linear dynamical systems, the dynamics of agent $i$ is described by

$$
\begin{gathered}
D^{\alpha} x_{i}(t)=A x_{i}(t)+B u_{i}(t) \\
y_{i}(t)=C x_{i}(t) \quad(i=1,2, \ldots, N),
\end{gathered}
$$

where $0<\alpha<1$ is the fractional order, $x_{i}(t)=\left(x_{i 1}(t), x_{i 2}(t), \ldots, x_{i n}(t)\right) \in \mathbb{R}^{n}$ is position state vector of $i$ th agent, $u_{i}(t)=\left(u_{i 1}(t), u_{i 2}(t), \ldots, u_{i m}(t)\right) \in \mathbb{R}^{m}$ is the control input, and $y_{i}(t)=$ $\left(y_{i 1}(t), y_{i 2}(t), \ldots, y_{i p}(t)\right) \in \mathbb{R}^{p}$ is the measured output. $A, B$, and $C$ are some real matrices with compatible dimensions, and

$$
A=A_{0}+\Delta A(t), \quad B=B_{0}+\Delta B(t)
$$

where $\Delta A(t)$ and $\Delta B(t)$ represent the parameter uncertainties satisfying the following conditions:

$$
\Delta A(t)=D_{A} F_{A}(t) E_{A}, \quad \Delta B(t)=D_{B} F_{B}(t) E_{B},
$$

and $D_{A}, E_{A}, D_{B}$, and $E_{B}$ are some constant matrices with appropriate dimensions, $F_{A}(t)$ and $F_{B}(t)$ are the uncertainties satisfying $F_{A}^{T}(t) F_{A}(t) \leq I, F_{B}^{T}(t) F_{B}(t) \leq I$, in which $I \in \mathbb{R}^{n \times n}$ denotes the identity matrix.

Remark 2.1. In [9-11], observer-based protocols are proposed for consensus of linear multiagent systems with reduced order, where the multiagent systems are described by integer-order systems, uncertainty is not considered. But in (2.1), every agent is required with the same dynamics and uncertainties, which have certain conservation. In order to reduce this conservation, we can consider a more general multiagent systems:

$$
\begin{gathered}
D^{\alpha} x_{i}(t)=A_{i} x_{i}(t)+B_{i} u_{i}(t) \\
y_{i}(t)=C_{i} x_{i}(t) \quad(i=1,2, \ldots, N), \\
A_{i}=A_{i 0}+\Delta A_{i}(t), \quad B_{i}=B_{i 0}+\Delta B_{i}(t),
\end{gathered}
$$

where $\Delta A_{i}(t)$ and $\Delta B_{i}(t)$ represent the parameter uncertainties of $i$ th agent satisfying the following conditions:

$$
\Delta A_{i}(t)=D_{A i} F_{A i}(t) E_{A i}, \quad \Delta B_{i}(t)=D_{B i} F_{B i}(t) E_{B i},
$$

for the above model, we can get similar results, but the mathematical treatment becomes more complicated, the important and meaningful research topics will be considered for our future research. 
Based on the design idea of the observer, a Luenberger-type fractional-order linear observer is constructed as follows:

$$
D^{\alpha} \widehat{x}_{i}(t)=A \widehat{x}_{i}(t)+B u_{i}(t)+K_{2}\left(y_{i}(t)-\widehat{y}_{i}(t)\right) \widehat{y}_{i}(t)=C \widehat{x}_{i}(t) \quad(i=1,2, \ldots, N),
$$

where $\widehat{x}_{i}(t)$ and $\widehat{y}_{i}(t)$ are the state and the output of observer. $K_{2} \in \mathbb{R}^{n \times p}$ is a feedback gain matrix to be determined later.

Similar to [26], observer-type consensus protocol based on the relative observer states between neighboring agents is given as

$$
u_{i}(t)=K_{1} \sum_{j \in N_{i}} a_{i j}\left(\widehat{x}_{i}(t)-\widehat{x}_{j}(t)\right)=-\sum_{j \in N_{i}} l_{i j} K_{1} \widehat{x}_{j}(t) \quad(i=1,2, \ldots, N)
$$

where $K_{1} \in \mathbb{R}^{p \times n}$ is the feedback gain matrix to be designed.

Remark 2.2. It should be noticed that the consensus protocol (2.14) is based on the relative states of the neighboring observers. To our best knowledge, it is novel for fractional-order multiagent systems.

Substituting (2.14) into (2.8), and (2.13) respectively, and using Kronecker product, (2.8) and (2.13) can be rewritten in the following compact form:

$$
\begin{gathered}
D^{\alpha} x(t)=\left(I_{N} \otimes A\right) x(t)-\left(L \otimes B K_{1}\right) \widehat{x}(t), \\
y(t)=\left(I_{N} \otimes C\right) x(t), \\
D^{\alpha} \widehat{x}(t)=\left(I_{N} \otimes A-L \otimes B K_{1}\right) \widehat{x}(t)+\left(I_{N} \otimes K_{2} C\right)(x(t)-\widehat{x}(t)), \\
\widehat{y}(t)=\left(I_{N} \otimes C\right) \widehat{x}(t),
\end{gathered}
$$

Let $e(t)=x(t)-\widehat{x}(t)$, the closed-loop system is given by

$$
\begin{gathered}
D^{\alpha} \widehat{x}(t)=\left(I_{N} \otimes A-L \otimes B K_{1}\right) \widehat{x}(t)+\left(I_{N} \otimes K_{2} C\right) e(t) \\
D^{\alpha} e(t)=\left(I_{N} \otimes A-I_{N} \otimes K_{2} C\right) e(t)
\end{gathered}
$$

which can be rewritten as

$$
D^{\alpha} X(t)=A_{K} X(t),
$$

where

$$
X(t)=\left[\begin{array}{l}
\widehat{x}(t) \\
e(t)
\end{array}\right], \quad A_{K}=\left[\begin{array}{cc}
I_{N} \otimes A-L \otimes B K_{1} & I_{N} \otimes K_{2} C \\
0 & I_{N} \otimes A-I_{N} \otimes K_{2} C
\end{array}\right] .
$$

In the following, an LMI-based design method is developed for the consensus of a class of linear fractional-order uncertain multiagents. Before giving the main results, the following definition and lemmas are introduced. 
Definition 2.3 (see [26]). The consensus problem of multiagent systems (2.8) is solved by protocol (2.14) if the states of (2.8) satisfy

$$
\lim _{t \rightarrow \infty}\left\|x_{i}(t)-x_{j}(t)\right\|=0 \quad(i=1,2, \ldots, N)
$$

Lemma 2.4 (see [32]). Let $a \in \mathbb{R}$ and $A, B, C, D$ be matrices with appropriate dimensions. The following properties can be proved by the definition of Kronecker product:

$$
\begin{aligned}
& \text { (1) } a(A \otimes B)=(a A) \otimes B=A \otimes(a B) \\
& \text { (2) }(A \otimes B)^{T}=A^{T} \otimes B^{T} \\
& \text { (3) }(A \otimes B)(C \otimes D)=(A C) \otimes(B D) \\
& \text { (4) } A \otimes B \otimes C=(A \otimes B) \otimes C=A \otimes(B \otimes C) .
\end{aligned}
$$

Lemma 2.5 (see [33]). Let $A \in \mathbb{R}^{n \times n}$ be a real matrix, a necessary and sufficient condition for the asymptotical stability of $D^{\alpha} x(t)=A x(t)$ is

$$
|\arg (\operatorname{spec}(A))|>\frac{\alpha \pi}{2}
$$

where $\operatorname{spec}(A)$ is the spectrum of all eigenvalues of $A$, fractional order satisfying $0<\alpha<2$.

Lemma 2.6 (see [18]). Let $A \in \mathbb{R}^{n \times n}$ and $0<\alpha<1$, then the fractional-order system $D^{\alpha} x(t)=$ $A x(t)$ is asymptotically stable if and only if there exist two real symmetric positive definite matrices $P_{k 1} \in \mathbb{R}^{n \times n}(k=1,2)$ and two skew-symmetric matrices $P_{k 2} \in \mathbb{R}^{n \times n}(k=1,2)$, such that

$$
\begin{gathered}
\sum_{i=1}^{2} \sum_{j=1}^{2} \operatorname{sym}\left\{\theta_{i j} \otimes\left(A P_{i j}\right)\right\}<0, \\
{\left[\begin{array}{cc}
P_{11} & P_{12} \\
-P_{12} & P_{11}
\end{array}\right]>0 \quad\left[\begin{array}{cc}
P_{21} & P_{22} \\
-P_{22} & P_{21}
\end{array}\right]>0,}
\end{gathered}
$$

where

$$
\begin{aligned}
\theta_{11} & =\left[\begin{array}{cc}
\sin \theta & -\cos \theta \\
\cos \theta & \sin \theta
\end{array}\right], & \theta_{12} & =\left[\begin{array}{cc}
\cos \theta & \sin \theta \\
-\sin \theta & \cos \theta
\end{array}\right], \\
\theta_{21} & =\left[\begin{array}{cc}
\sin \theta & \cos \theta \\
-\cos \theta & \sin \theta
\end{array}\right], & \theta_{22} & =\left[\begin{array}{cc}
-\cos \theta & \sin \theta \\
-\sin \theta & -\cos \theta
\end{array}\right],
\end{aligned}
$$

and $\theta=\alpha \pi / 2$.

Lemma 2.7 (see[34]). Let $H$ and $E$ be real matrices of appropriate dimensions with $F(t)$ satisfying $F^{T}(t) F(t)<I$, and there exists positive scalar $\varepsilon>0$, such that

$$
H F(t) E+E^{T} F^{T}(t) H^{T}<\varepsilon H H^{T}+\varepsilon^{-1} E^{T} E .
$$


Recall that for any matrix $\Pi \in \mathbb{R}^{m \times n}$ with full row rank, there exists a singular value decomposition of $\Pi$ as follows:

$$
\Pi=U\left[\begin{array}{ll}
S & 0
\end{array}\right] V^{T},
$$

where $S \in \mathbb{R}^{m \times p}$ is a diagonal matrix with positive elements in decreasing order, $p=\min \{m, n\}$, $U \in \mathbb{R}^{p \times p}$ and $V \in \mathbb{R}^{n \times n}$ are unitary matrices. Then the following, lemma holds.

Lemma 2.8 (see [19]). Given matrix $\Pi \in \mathbb{R}^{m \times n}$ with $\operatorname{rank}(\Pi)=p$, assume that $X \in \mathbb{R}^{n \times n}$ is a symmetric matrix, there exists a matrix $\bar{X} \in \mathbb{R}^{m \times n}$ satisfying $\Pi X=\bar{X} \Pi$ if and only if $X$ can be expressed as

$$
X=V\left[\begin{array}{cc}
X_{11} & 0 \\
0 & X_{22}
\end{array}\right] V^{T}
$$

where $X_{11} \in \mathbb{R}^{m \times m}$ and $X_{22} \in \mathbb{R}^{(n-m) \times(n-m)}$.

Remark 2.9. As we known, the existing results about stability of fractional-order deterministic systems are based on Lemma 2.5, which cannot be applied directing to fractional-order uncertain systems, because this method needs to compute all eigenvalues of system (2.17), it is hard to yield all eigenvalues for fractional-order systems with uncertain parameters, the paper can effectively avoid this difficulty by using Lemma 2.6.

\section{Main Results}

In this section, a sufficient condition is first derived for robust asymptotic stability of uncertain fractional-order linear systems (2.17), based on the stability criterion, an LMIbased approach is proposed for designing observer-type consensus protocol for the uncertain fractional-order multiagent systems (2.8).

Theorem 3.1. For given two feedback gain matrix $K_{1} \in \mathbb{R}^{p \times n}$ and $K_{2} \in \mathbb{R}^{n \times p}$, the uncertain fractional-order multiagent systems (2.17) are asymptotically stable if there exist two positive matrices $Q_{1} \in \mathbb{R}^{n \times n}, Q_{2} \in \mathbb{R}^{n \times n}$ and four real positive scalars $a_{1}, a_{2}, b_{1}$, and $b_{2}$ such that the following linear matrix inequality holds:

$$
\left[\begin{array}{ccccc}
\Pi_{1} & * & * & * & * \\
\Pi_{2} & -a_{1} \otimes I_{4 N n} & * & * & * \\
\Pi_{2} & 0 & -a_{2} \otimes I_{4 N n} & * & * \\
\Pi_{3} & 0 & 0 & -b_{1} \otimes I_{4 N n} & * \\
\Pi_{3} & 0 & 0 & 0 & -b_{2} \otimes I_{4 N n}
\end{array}\right]<0,
$$

where

$$
\Pi_{1}=\left[\begin{array}{cccc}
\Pi_{11} & * & * & * \\
\Pi_{21} & \Pi_{22} & * & * \\
0 & 0 & \Pi_{11} & * \\
0 & 0 & \Pi_{21} & \Pi_{22}
\end{array}\right]
$$




$$
\begin{aligned}
\Pi_{2}= & {\left[\begin{array}{cccc}
I_{N} \otimes E_{A} Q_{1} & 0 & 0 & 0 \\
0 & I_{N} \otimes E_{A} Q_{2} & 0 & 0 \\
0 & 0 & I_{N} \otimes E_{A} Q_{1} & 0 \\
0 & 0 & 0 & I_{N} \otimes E_{A} Q_{2}
\end{array}\right], } \\
\Pi_{3}= & {\left[\begin{array}{cccc}
I_{N} \otimes E_{B} K_{1} Q_{1} & 0 & 0 & 0 \\
0 & 0 & 0 & 0 \\
0 & 0 & I_{N} \otimes E_{B} K_{1} Q_{1} & 0 \\
0 & 0 & 0 & 0
\end{array}\right], } \\
\Pi_{11}= & 2\left(I_{N} \otimes A_{0} Q_{1}+I_{N} \otimes Q_{1} A_{0}^{T}-L \otimes B_{0} K_{1} Q_{1}-L^{T} \otimes Q_{1} K_{1}^{T} B_{0}^{T}\right) \sin \theta \\
& +\left(a_{1}+a_{2}\right) I_{N} \otimes D_{A} D_{A}^{T}+\left(b_{1}+b_{2}\right) L L^{T} \otimes D_{B} D_{B^{\prime}}^{T} \\
\Pi_{21}= & 2\left(I_{N} \otimes Q_{2} C^{T} K_{2}^{T}\right) \sin \theta \\
\Pi_{22}= & 2\left(I_{N} \otimes A_{0} Q_{2}+I_{N} \otimes Q_{2} A_{0}^{T}-I_{N} \otimes K_{2} C Q_{2}-I_{N} \otimes Q_{2} C^{T} K_{2}^{T}\right) \sin \theta+\left(a_{1}+a_{2}\right) \otimes D_{A} D_{A}^{T} .
\end{aligned}
$$

Proof. It follows from Lemma 2.6 that the system (2.17) is asymptotically stable if there exist two real symmetric positive definite matrices $P_{k 1} \in \mathbb{R}^{2 n \times 2 n}(k=1,2)$ and two skew-symmetric matrices $P_{k 2} \in \mathbb{R}^{2 n \times 2 n}(k=1,2)$ such that the following linear matrix inequality holds:

$$
\Pi=\sum_{i=1}^{2} \sum_{j=1}^{2} \operatorname{sym}\left\{\theta_{i j} \otimes\left(A_{K} P_{i j}\right)\right\}<0 .
$$

Setting $P_{11}=P_{21}=Q=\operatorname{diag}\left\{I_{N} \otimes Q_{1}, I_{N} \otimes Q_{2}\right\}$ and $P_{12}=P_{22}=0$, then (3.3) will degenerate to the following inequality:

$$
\Pi=\sum_{i=1}^{2} \operatorname{sym}\left\{\theta_{i 1} \otimes\left(A_{K} Q\right)\right\}<0,
$$

then system (2.17) is asymptotically stable. By simple calculation, the following equality holds:

$$
\begin{aligned}
A_{K} Q= & {\left[\begin{array}{cc}
I_{N} \otimes A_{0} Q_{1}-L \otimes B_{0} K_{1} Q_{1} & I_{N} \otimes K_{2} C Q_{2} \\
0 & I_{N} \otimes A_{0} Q_{2}-I_{N} \otimes K_{2} C Q_{2}
\end{array}\right] } \\
& +\left[\begin{array}{cc}
I_{N} \otimes D_{A} F_{A}(t) E_{A} Q_{1} & 0 \\
0 & I_{N} \otimes D_{A} F_{A}(t) E_{A} Q_{2}
\end{array}\right]+\left[\begin{array}{cc}
-L \otimes D_{B} F_{B}(t) E_{B} K_{1} Q_{1} & 0 \\
0 & 0
\end{array}\right] .
\end{aligned}
$$


For the convenience of later analysis, we denote

$$
\begin{gathered}
\Delta_{1}=\left[\begin{array}{cc}
I_{N} \otimes A_{0} Q_{1}-L \otimes B_{0} K_{1} Q_{1} & I_{N} \otimes K_{2} C Q_{2} \\
0 & I_{N} \otimes A_{0} Q_{2}-I_{N} \otimes K_{2} C Q_{2}
\end{array}\right], \\
\Delta_{2}=\left[\begin{array}{cc}
I_{N} \otimes D_{A} F_{A}(t) E_{A} Q_{1} & 0 \\
0 & I_{N} \otimes D_{A} F_{A}(t) E_{A} Q_{2}
\end{array}\right], \\
\Delta_{3}=\left[\begin{array}{cc}
-L \otimes D_{B} F_{B}(t) E_{B} K_{1} Q_{1} & 0 \\
0 & 0
\end{array}\right] .
\end{gathered}
$$

We rewrite (3.5) as follows:

$$
\begin{aligned}
\Pi & =\sum_{i=1}^{2} \operatorname{sym}\left\{\theta_{i 1} \otimes\left(A_{K} Q\right)\right\} \\
& =\sum_{i=1}^{2} \operatorname{sym}\left\{\theta_{i 1} \otimes \Delta_{1}\right\}+\sum_{i=1}^{2} \operatorname{sym}\left\{\theta_{i 1} \otimes \Delta_{2}\right\}+\sum_{i=1}^{2} \operatorname{sym}\left\{\theta_{i 1} \otimes \Delta_{3}\right\} .
\end{aligned}
$$

Based on Lemma 2.6, it follows from (3.5) that

$$
\begin{aligned}
\sum_{i=1}^{2} \operatorname{sym}\left\{\theta_{i 1} \otimes \Delta_{1}\right\} & =\operatorname{sym}\left\{\left[\begin{array}{cc}
2 \sin \theta & 0 \\
0 & 2 \sin \theta
\end{array}\right] \otimes \Delta_{1}\right\} \\
& =\left[\begin{array}{cccc}
\Pi_{11}-\Phi_{1} & * & * & * \\
\Pi_{21} & \Pi_{22}-\Phi_{2} & * & * \\
0 & 0 & \Pi_{11}-\Phi_{1} & * \\
0 & 0 & \Pi_{21} & \Pi_{22}-\Phi_{2}
\end{array}\right],
\end{aligned}
$$

where

$$
\begin{aligned}
& \Phi_{1}=\left(a_{1}+a_{2}\right) \otimes D_{A} D_{A}^{T}+\left(b_{1}+b_{2}\right) L L^{T} \otimes D_{B} D_{B^{\prime}}^{T} \\
& \Phi_{2}=\left(a_{1}+a_{2}\right) \otimes D_{A} D_{A}^{T} .
\end{aligned}
$$

Note that $\theta_{i 1} \theta_{i 1}^{T}=I_{2}(i=1,2)$, for any real positive scalars $a_{1}$ and $a_{2}$, it follows from (3.9) and Lemma 2.7 that

$$
\begin{aligned}
& \sum_{i=1}^{2} \operatorname{sym}\left\{\theta_{i 1} \otimes \Delta_{2}\right\} \\
& \quad=\sum_{i=1}^{2} \operatorname{sym}\left\{\theta_{i 1} \otimes\left\{I_{N} \otimes\left[\begin{array}{cc}
D_{A} & 0 \\
0 & D_{A}
\end{array}\right]\left[\begin{array}{cc}
F_{A}(t) & 0 \\
0 & F_{A}(t)
\end{array}\right]\left[\begin{array}{cc}
E_{A} Q_{1} & 0 \\
0 & E_{A} Q_{2}
\end{array}\right]\right\}\right.
\end{aligned}
$$




$$
\begin{aligned}
& =\sum_{i=1}^{2} \operatorname{sym}\left\{\theta_{i 1} \otimes\left\{\left[\begin{array}{cc}
I_{N} \otimes D_{A} & 0 \\
0 & I_{N} \otimes D_{A}
\end{array}\right]\left[\begin{array}{cc}
I_{N} \otimes F_{A}(t) & 0 \\
0 & I_{N} \otimes F_{A}(t)
\end{array}\right]\left[\begin{array}{cc}
I_{N} \otimes E_{A} Q_{1} & 0 \\
0 & I_{N} \otimes E_{A} Q_{2}
\end{array}\right]\right\}\right\} \\
& =\sum_{i=1}^{2} \operatorname{sym}\left\{\left(\theta_{i 1} \otimes\left[\begin{array}{cc}
I_{N} \otimes D_{A} & 0 \\
0 & I_{N} \otimes D_{A}
\end{array}\right]\right)\left(I_{2} \otimes\left[\begin{array}{cc}
I_{N} \otimes F_{A}(t) & 0 \\
0 & I_{N} \otimes F_{A}(t)
\end{array}\right]\right)\right. \\
& \left.\times\left(I_{2} \otimes\left[\begin{array}{cc}
I_{N} \otimes E_{A} Q_{1} & 0 \\
0 & I_{N} \otimes E_{A} Q_{2}
\end{array}\right]\right)\right\} \\
& \leq \sum_{i=1}^{2}\left\{a_{i}\left(\theta_{i 1} \otimes\left[\begin{array}{cc}
I_{N} \otimes D_{A} & 0 \\
0 & I_{N} \otimes D_{A}
\end{array}\right]\right)\left(\theta_{i 1} \otimes\left[\begin{array}{cc}
I_{N} \otimes D_{A} & 0 \\
0 & I_{N} \otimes D_{A}
\end{array}\right]\right)^{T}\right. \\
& \left.+a_{i}^{-1}\left(I_{2} \otimes\left[\begin{array}{cc}
I_{N} \otimes D_{A} Q_{1} & 0 \\
0 & I_{N} \otimes D_{A} Q_{2}
\end{array}\right]\right)^{T}\left(I_{2} \otimes\left[\begin{array}{cc}
I_{N} \otimes D_{A} Q_{1} & 0 \\
0 & I_{N} \otimes D_{A} Q_{2}
\end{array}\right]\right)\right\} \\
& =\sum_{i=1}^{2}\left\{a_{i}\left(I_{2} \otimes\left[\begin{array}{cc}
I_{N} \otimes D_{A} D_{A}^{T} & 0 \\
0 & I_{N} \otimes D_{A} D_{A}^{T}
\end{array}\right]\right)\right. \\
& \left.+a_{i}^{-1}\left(I_{2} \otimes\left[\begin{array}{cc}
I_{N} \otimes D_{A} Q_{1} & 0 \\
0 & I_{N} \otimes D_{A} Q_{2}
\end{array}\right]\right)^{T}\left(I_{2} \otimes\left[\begin{array}{cc}
I_{N} \otimes D_{A} Q_{1} & 0 \\
0 & I_{N} \otimes D_{A} Q_{2}
\end{array}\right]\right)\right\} .
\end{aligned}
$$

By the same argument, for any real positive scalars $b_{1}$ and $b_{2}$, one has

$$
\begin{aligned}
& \sum_{i=1}^{2} \operatorname{sym}\left\{\theta_{i 1} \otimes \Delta_{3}\right\} \\
& \leq \sum_{i=1}^{2}\left\{b_{i}\left(I_{2} \otimes\left[\begin{array}{cc}
L L^{T} \otimes D_{B} D_{B}^{T} & 0 \\
0 & 0
\end{array}\right]\right)\right. \\
& \left.\quad+b_{i}^{-1}\left(I_{2} \otimes\left[\begin{array}{cc}
I_{N} \otimes E_{B} K_{1} Q_{1} & 0 \\
0 & 0
\end{array}\right]\right)\left(I_{2} \otimes\left[\begin{array}{cc}
I_{N} \otimes E_{B} K_{1} Q_{1} & 0 \\
0 & 0
\end{array}\right]\right)\right\}
\end{aligned}
$$

Combined with (3.10)-(3.13), we can obtain

$$
\begin{aligned}
& \Pi \leq \operatorname{sym}\left\{\left[\begin{array}{cc}
2 \sin (\theta) & 0 \\
0 & 2 \sin (\theta)
\end{array}\right] \otimes \Delta_{1}\right\}+\sum_{i=1}^{2}\left\{a_{i}\left(I_{2} \otimes\left[\begin{array}{cc}
I_{N} \otimes D_{A} D_{A}^{T} & 0 \\
0 & I_{N} \otimes D_{A} D_{A}^{T}
\end{array}\right]\right)\right\} \\
& +\sum_{i=1}^{2}\left\{b_{i}\left(I_{2} \otimes\left[\begin{array}{ccc}
L L^{T} \otimes D_{B} D_{B}^{T} & 0 \\
0 & 0
\end{array}\right]\right)\right\} \\
& +\sum_{i=1}^{2} a_{i}^{-1}\left(I_{2} \otimes\left[\begin{array}{cc}
I_{N} \otimes D_{A} Q_{1} & 0 \\
0 & I_{N} \otimes D_{A} Q_{2}
\end{array}\right]\right)^{T}\left(I_{2} \otimes\left[\begin{array}{cc}
I_{N} \otimes D_{A} Q_{1} & 0 \\
0 & I_{N} \otimes D_{A} Q_{2}
\end{array}\right]\right) \\
& +\sum_{i=1}^{2} b_{i}^{-1}\left(I_{2} \otimes\left[\begin{array}{cc}
I_{N} \otimes E_{B} K_{1} Q_{1} & 0 \\
0 & 0
\end{array}\right]\right)^{T}\left(I_{2} \otimes\left[\begin{array}{cc}
I_{N} \otimes E_{B} K_{1} Q_{1} & 0 \\
0 & 0
\end{array}\right]\right) \text {, }
\end{aligned}
$$


we can immediately obtain (3.14) from (3.1) by using the Schur complement. This completes the proof.

Remark 3.2. If $K_{1}$ and $K_{2}$ are not given beforehand, owing to the existence of the nonlinear terms such as $K_{1} Q_{1}, K_{2} C Q_{2}$, the matrix inequality (3.1) is not an LMI. However, applying Lemma 2.8, it can be transformed into an LMI, and the main results are given in the following theorem.

Theorem 3.3. Assume that singular value decomposition of output matrix $C$ with full-row rank is $C=U\left[\begin{array}{ll}S & 0\end{array}\right] V^{T}$, then the closed-loop control system (2.17) under the observer-type consensus protocol (2.14) is robust asymptotically stable, if there exist symmetric positive matrices $Q_{1} \in \mathbb{R}^{n \times n}$, $Q_{11} \in \mathbb{R}^{p \times p}, Q_{22} \in \mathbb{R}^{(n-p) \times(n-p)}$ and two matrices $X_{1} \in \mathbb{R}^{p \times n}, X_{2} \in \mathbb{R}^{n \times p}$, and four real positive scalars $a_{1}, a_{2}, b_{1}$, and $b_{2}$ such that the following linear matrix inequality holds:

$$
\left[\begin{array}{ccccc}
\bar{\Pi}_{1} & * & * & * & * \\
\Pi_{2} & -a_{1} \otimes I_{4 N n} & * & * & * \\
\Pi_{2} & 0 & -a_{2} \otimes I_{4 N n} & * & * \\
\bar{\Pi}_{3} & 0 & 0 & -b_{1} \otimes I_{4 N n} & * \\
\bar{\Pi}_{3} & 0 & 0 & 0 & -b_{2} \otimes I_{4 N n}
\end{array}\right]<0,
$$

where

$$
\begin{aligned}
& \bar{\Pi}_{1}=\left[\begin{array}{cccc}
\bar{\Pi}_{11} & * & * & * \\
\bar{\Pi}_{21} & \bar{\Pi}_{22} & * & * \\
0 & 0 & \bar{\Pi}_{11} & * \\
0 & 0 & \bar{\Pi}_{21} & \bar{\Pi}_{22}
\end{array}\right], \\
& \bar{\Pi}_{3}=\left[\begin{array}{cccc}
I_{N} \otimes E_{B} X_{1} & 0 & 0 & 0 \\
0 & 0 & 0 & 0 \\
0 & 0 & I_{N} \otimes E_{B} X_{1} & 0 \\
0 & 0 & 0 & 0
\end{array}\right], \\
& \bar{\Pi}_{11}=2\left(I_{N} \otimes A_{0} Q_{1}+I_{N} \otimes Q_{1} A_{0}^{T}-L \otimes B_{0} X_{1}-L^{T} \otimes X_{1}^{T} B_{0}^{T}\right) \sin \theta \\
& +\left(a_{1}+a_{2}\right) I_{N} \otimes D_{A} D_{A}^{T}+\left(b_{1}+b_{2}\right) L L^{T} \otimes D_{B} D_{B^{\prime}}^{T} \\
& \bar{\Pi}_{21}=2\left(I_{N} \otimes C^{T} X_{2}^{T}\right) \sin \theta, \\
& \bar{\Pi}_{22}=2\left(I_{N} \otimes A_{0} Q_{2}+I_{N} \otimes Q_{2} A_{0}^{T}-I_{N} \otimes X_{2} C-I_{N} \otimes C^{T} X_{2}^{T}\right) \sin \theta+\left(a_{1}+a_{2}\right) \otimes D_{A} D_{A}^{T} \text {, } \\
& Q_{2}=V\left[\begin{array}{cc}
Q_{11} & 0 \\
0 & Q_{22}
\end{array}\right] V^{T},
\end{aligned}
$$

then the feedback gain matrices are given by

$$
K_{1}=X_{1} Q_{1}^{-1}, \quad K_{2}=X_{2} U S Q_{11}^{-1} S^{-1} U^{-1}
$$


Proof. Since $C=U\left[\begin{array}{ll}S & 0\end{array}\right] V^{T}$ and $Q_{2}=V\left[\begin{array}{cc}Q_{11} & 0 \\ 0 & Q_{22}\end{array}\right] V^{T}$, from Lemma 2.5, there exists $\bar{Q}_{2}$, such that $C Q_{2}=\bar{Q}_{2} C$, where $\bar{Q}_{2}=U S Q_{11} S^{-1} U^{-1}$, it is easy derived that $\bar{Q}_{2}^{-1}=U S Q_{11}^{-1} S^{-1} U^{-1}$. Setting $K_{1} Q_{1}=X_{1}$ and $K_{2} \bar{Q}_{2}=X_{2}$, and (3.1) is inequivalent to (3.15). Moreover, the feedback gain matrices are obtained by

$$
\begin{aligned}
& K_{1}=X_{1} Q_{1}^{-1}, \\
& K_{2}=X_{2} \bar{Q}_{2}^{-1}=X_{2} U S Q_{11}^{-1} S^{-1} U^{-1} .
\end{aligned}
$$

Remark 3.4. Linear matrix inequality technique has attracted much more attention and has wide applications because of its high performance in analysis and design in the control systems, which in particular has a better advantage in dealing with uncertain systems. Noting that Theorem 3.3 provides an LMI-based method of designing feed-back gain matrices, that is, $K_{1}$ and $K_{2}$ can easily be obtained by LMI toolbox.

In particular, if the position state of multiagent can be obtained, then we can select the following consensus protocol:

$$
u_{i}(t)=-\sum_{j \in N_{i}} l_{i j} K x_{j}(t) \quad(i=1,2 \ldots, N),
$$

substituting (3.19) into (2.8), we have

$$
\begin{gathered}
D^{\alpha} x(t)=\left(I_{N} \otimes A-L \otimes B K\right) x(t), \\
y(t)=\left(I_{N} \otimes C\right) x(t) .
\end{gathered}
$$

Based on (3.20), the following consensus criteria can derived without proof.

Corollary 3.5. The fractional-order uncertain multiagent systems (3.20) can achieve consensus by protocol (3.19) if there exist symmetric positive matrix $Q \in \mathbb{R}^{n \times n}$ and a matrix $X \in \mathbb{R}^{p \times n}$, and four real-positive scalars $a_{1}, a_{2}, b_{1}$, and $b_{2}$, such that

$$
\left[\begin{array}{ccccc}
\hat{\Pi}_{1} & * & * & * & * \\
\widehat{\Pi}_{2} & -a_{1} \otimes I_{2 N n} & * & * & * \\
\widehat{\Pi}_{2} & 0 & -a_{2} \otimes I_{2 N n} & * & * \\
\widehat{\Pi}_{3} & 0 & 0 & -b_{1} \otimes I_{2 N n} & * \\
\widehat{\Pi}_{3} & 0 & 0 & 0 & -b_{2} \otimes I_{2 N n}
\end{array}\right]<0,
$$

where

$$
\widehat{\Pi}_{1}=\left[\begin{array}{cc}
\widehat{\Pi}_{11} & * \\
0 & \widehat{\Pi}_{11}
\end{array}\right]
$$




$$
\begin{aligned}
\widehat{\Pi}_{2} & =\left[\begin{array}{cc}
I_{N} \otimes E_{A} Q & 0 \\
0 & I_{N} \otimes E_{A} Q
\end{array}\right], \\
\widehat{\Pi}_{3} & =\left[\begin{array}{cc}
I_{N} \otimes E_{B} X & 0 \\
0 & I_{N} \otimes E_{A} X
\end{array}\right], \\
\widehat{\Pi}_{11} & =2\left(I_{N} \otimes A_{0} Q+I_{N} \otimes Q A_{0}^{T}-L \otimes B_{0} X-L^{T} \otimes X^{T} B_{0}^{T}\right) \sin \theta,
\end{aligned}
$$

then the feedback gain matrix is given as follows:

$$
K=X Q^{-1}
$$

\section{A Numerical Example}

In this section, a numerical example is given to verify the effectiveness of proposed observertype consensus protocol in the preceding section.

Example 4.1. Consider fractional-order uncertain multiagent systems consisting of four agents, the interaction diagraph is shown in Figure 1 with the weights on the connections. The related parameters are given as follows:

$$
\begin{aligned}
A_{0} & =\left[\begin{array}{ccc}
-0.4 & 0.35 & -0.65 \\
-0.9 & -2.7 & 1.1 \\
-0.5 & -1.35 & -2.25
\end{array}\right], \quad B_{0}=\left[\begin{array}{l}
1.45 \\
0.75 \\
0.75
\end{array}\right] \quad C=\left[\begin{array}{c}
1.5 \\
2 \\
1
\end{array}\right]^{T}, \\
D_{A} & =\left[\begin{array}{ccc}
-0.1 & 0.05 & 0.1 \\
-0.1 & -0.3 & 0.1 \\
-0.15 & -0.08 & -0.4
\end{array}\right], \quad E_{A}=\left[\begin{array}{lll}
1 & 0 & 0 \\
0 & 1 & 0 \\
0 & 0 & 1
\end{array}\right], \quad D_{B}=\left[\begin{array}{ccc}
-0.01 & 0.05 & 0.01 \\
-0.15 & -0.08 & -0.04 \\
0 & 0 & 1
\end{array}\right], \\
E_{B} & =\left[\begin{array}{c}
0.1 \\
0.15 \\
0.15
\end{array}\right], \quad F_{A}(t)=F_{B}(t)=\left[\begin{array}{ccc}
\sin (0.1 \pi t) & 0 & 0 \\
0 & \cos (0.1 \pi t) & 0 \\
0 & 0 & \sin (0.1 \pi t)
\end{array}\right] .
\end{aligned}
$$

For given fractional-order $\alpha=0.9$, by using the Matlab LMI toolbox in Theorem 3.3, the feasible solution (3.15) is given as follows:

$$
\begin{aligned}
& Q_{1}=\left[\begin{array}{ccc}
6.8316 & -2.7412 & -0.5239 \\
-2.7412 & 5.3247 & 0.6660 \\
-0.5237 & 0.6660 & 5.2857
\end{array}\right], \quad Q_{11}=11.7744, \quad Q_{22}=\left[\begin{array}{ll}
8.4600 & 1.9039 \\
1.9039 & 6.1330
\end{array}\right] \\
& X_{1}=\left[\begin{array}{lll}
0.0701 & -0.0483 & -0.1461
\end{array}\right], \quad X_{2}=\left[\begin{array}{lll}
0.8652 & -2.9990 & -2.7827
\end{array}\right]^{T}
\end{aligned}
$$

the gain matrices are given as follows:

$$
K_{1}=\left[\begin{array}{lll}
0.0075 & -0.0019 & -0.0267
\end{array}\right], \quad K_{2}=\left[\begin{array}{lll}
0.0735 & -0.2547 & -0.2363
\end{array}\right]^{T} .
$$




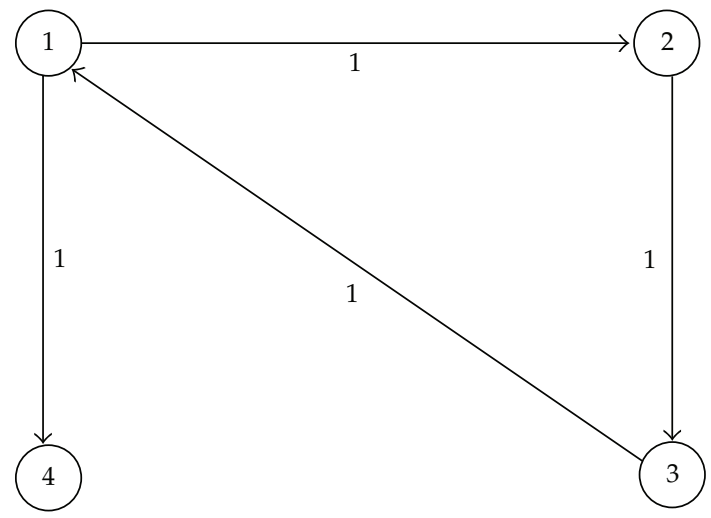

Figure 1: The topology structure of the agents.

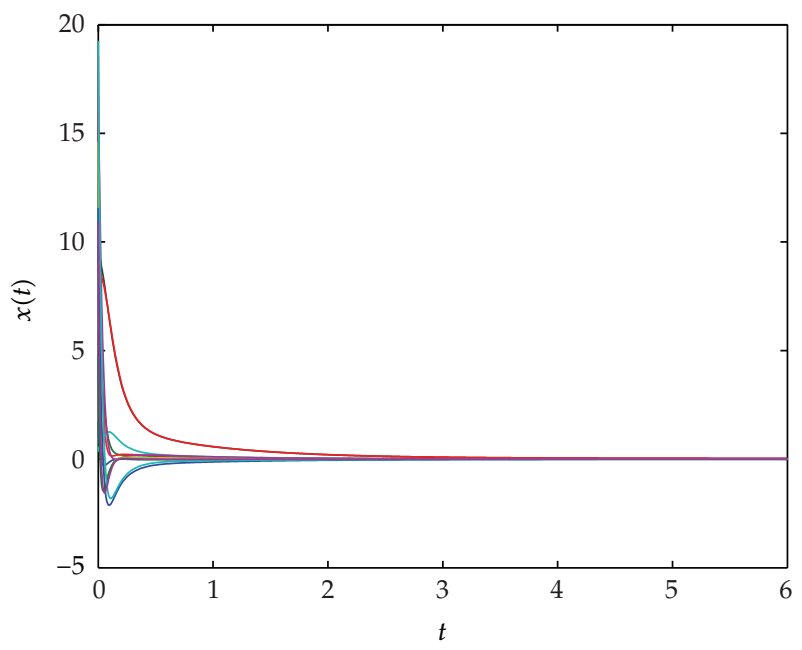

Figure 2: The position curves $x_{i}(t) \quad(i=1,2,3,4)$ for fractional-order $\alpha=0.9$.

In the numerical simulation, the position state curves of four multiagents are shown in Figure 2 with random initial state values, it can be seen that the consensus is achieved, and the response of the error $e(t)$ between the position state $x(t)$ and its estimate $\widehat{x}(t)$ are shown in Figure 3, which converge to zero. Therefore, the numerical simulation perfectly supports our theoretical results. To provided relatively complete information, the gain matrices are also listed in Table 1 for different fractional orders.

Remark 4.2. If fractional order $\alpha=1$, multiagent systems (2.8) reduce to integer-order multiagent systems as follows:

$$
\begin{gathered}
D x_{i}(t)=A x_{i}(t)+B u_{i}(t), \\
y_{i}(t)=C x_{i}(t) \quad(i=1,2, \ldots, N),
\end{gathered}
$$

similar to the process of Section 2, we can obtain the following augment system:

$$
D X(t)=A_{K} X(t) .
$$




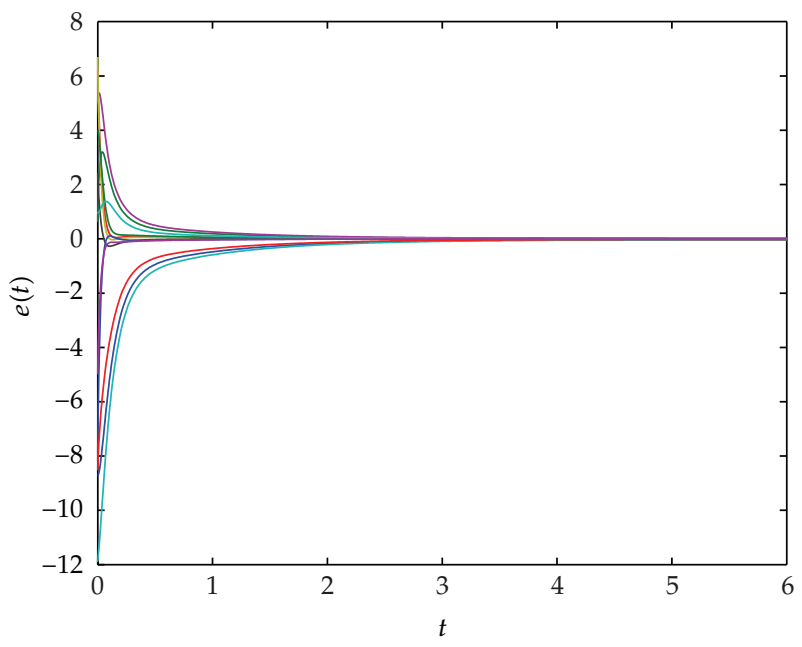

Figure 3: the error response $e(t)$ between the position $x(t)$ and its estimate $\widehat{x}(t)$ for fractional-order $\alpha=0.9$.

Table 1: Controller gain matrices $K_{1}$ and $K_{2}$ for different fractional order $\alpha$.

\begin{tabular}{|c|c|}
\hline Fractional orders $\alpha$ & Feedback gain matrices $K_{1}$ and $K_{2}$ \\
\hline 0.4 & $K_{1}=\left[\begin{array}{llll}0.0031 & -0.0136 & -0.0953\end{array}\right], K_{2}=\left[\begin{array}{lll}0.0137 & -0.2220 & -0.1835\end{array}\right]^{T}$ \\
\hline 0.5 & $K_{1}=\left[\begin{array}{lll}0.0173 & -0.0001 & -0.0160\end{array}\right], K_{2}=\left[\begin{array}{lll}0.0728 & -0.2379 & -0.2279\end{array}\right]^{T}$ \\
\hline 0.6 & $K_{1}=\left[\begin{array}{llll}0.0127 & -0.0006 & -0.0255\end{array}\right], K_{2}=\left[\begin{array}{lll}0.0686 & -0.2462 & -0.2304\end{array}\right]^{T}$ \\
\hline 0.7 & $K_{1}=[0.0097-0.0012-0.0281], K_{2}=\left[\begin{array}{lll}0.0691-0.2507 & -0.2326\end{array}\right]^{T}$ \\
\hline 0.8 & $K_{1}=[0.0082-0.0017-0.0275], K_{2}=\left[\begin{array}{lll}0.0716 & -0.2533-0.2348\end{array}\right]^{T}$ \\
\hline 0.9 & $K_{1}=[0.0075-0.0019-0.0267], K_{2}=\left[\begin{array}{lll}0.0735 & -0.2547-0.2363\end{array}\right]^{T}$ \\
\hline 0.99 & $K_{1}=\left[\begin{array}{lll}-0.0098 & -0.0118 & 0.0852\end{array}\right], K_{2}=\left[\begin{array}{lll}0.2423 & -0.1882 & -0.2746\end{array}\right]^{T}$ \\
\hline 0.999 & $K_{1}=\left[\begin{array}{lll}-0.0098 & -0.0118 & 0.0852\end{array}\right], K_{2}=\left[\begin{array}{lll}0.2423 & -0.1882 & -0.2746\end{array}\right]^{T}$ \\
\hline 0.9999 & $K_{1}=\left[\begin{array}{lll}-0.0098 & -0.0118 & 0.0852\end{array}\right], K_{2}=\left[\begin{array}{lll}0.2423 & -0.1882 & -0.2746\end{array}\right]^{T}$ \\
\hline$\alpha \rightarrow 1^{-}$ & $K_{1}=\left[\begin{array}{lll}-0.0098 & -0.0118 & 0.0852\end{array}\right], K_{2}=\left[\begin{array}{lll}0.2423 & -0.1882 & -0.2746\end{array}\right]^{T}$ \\
\hline
\end{tabular}

Construct the following Lyapunov function:

$$
V(t)=X^{T}(t) P X(t)
$$

where $P=\operatorname{diag}\left\{I_{N} \otimes P_{1}, I_{N} \otimes P_{2}\right\}$.

We can easily obtain similar results with Theorem 3.1 and Theorem 3.3, by using LMI toolbox in MATLAB, two unknown matrices can be obtained as follows:

$$
K_{1}=\left[\begin{array}{lll}
-0.0098 & -0.0118 & 0.0852
\end{array}\right], \quad K_{2}=\left[\begin{array}{lll}
0.2423 & -0.1882 & -0.2746
\end{array}\right]^{T},
$$

the results are consistent with corresponding fractional-order case in Table 1, when $\alpha \rightarrow 1^{-}$. 


\section{Conclusions}

The study investigates the consensus for a class of fractional-order uncertain multiagent systems. The consensus criteria are derived by applying the observer-type consensus protocol and stability theory of the fractional-order system, and these criteria are in the form of linear matrix inequalities which can be readily solved by applying the LMI toolbox. A numerical example is provided to demonstrate the validity of the presented consensus protocol.

\section{Acknowledgments}

This work was in part jointly supported by the National Science Foundation of China (nos. 61074024, 60874113), the Natural Science Foundation of Jiangsu Province of China (no. BK2010543), the Education Department Research Project of Zhejiang Province of China (no. Y201019013), and the Outstanding Young Teacher Project of Zhejiang Province.

\section{References}

[1] H. Yamaguchi, T. Arai, and G. Beni, "A distributed control scheme for multiple robotic vehicles to make group formations," Robotics and Autonomous Systems, vol. 36, no. 4, pp. 125-147, 2001.

[2] J. Desai, J. Ostrowski, and V. Kumar, "Modeling and control of formations of nonholonomic mobile robots," IEEE Transactions on Robotics and Automation, vol. 17, no. 6, pp. 905-908, 2001.

[3] J. Russell Carpenter, "Decentralized control of satellite formations," International Journal of Robust and Nonlinear Control, vol. 12, no. 2-3, pp. 141-161, 2002.

[4] R. Olfati-Saber and R. M. Murray, "Consensus problems in networks of agents with switching topology and time-delays," IEEE Transactions on Automatic Control, vol. 49, no. 9, pp. 1520-1533, 2004.

[5] W. Ren and R. W. Beard, "Consensus seeking in multiagent systems under dynamically changing interaction topologies," IEEE Transactions on Automatic Control, vol. 50, no. 5, pp. 655-661, 2005.

[6] W. Ren, R. Beard, and E. Atkins, "Information consensus in multivehicle cooperative control," IEEE Control Systems, vol. 27, no. 2, pp. 71-82, 2007.

[7] J. Zhu, Y.-P. Tian, and J. Kuang, "On the general consensus protocol of multi-agent systems with double-integrator dynamics," Linear Algebra and its Applications, vol. 431, no. 5-7, pp. 701-715, 2009.

[8] T. Li, M. Fu, L. Xie, and J.-F. Zhang, "Distributed consensus with limited communication data rate," IEEE Transactions on Automatic Control, vol. 56, no. 2, pp. 279-292, 2011.

[9] J. H. Seo, H. Shim, and J. Back, "Consensus of high-order linear systems using dynamic output feedback compensator: iow gain approach," Automatica, vol. 45, no. 11, pp. 2659-2664, 2009.

[10] T. Li and J.-F. Zhang, "Consensus conditions of multi-agent systems with time-varying topologies and stochastic communication noises," IEEE Transactions on Automatic Control, vol. 55, no. 9, pp. 2043-2057, 2010 .

[11] Z. Li, X. Liu, P. Lin, and W. Ren, "Consensus of linear multi-agent systems with reduced-order observer-based protocols," Systems E Control Letters, vol. 60, no. 7, pp. 510-516, 2011.

[12] R. Bagley and P. Torvik, "On the fractional calculus model of viscoelastic behavior," Journal of Rheology, vol. 30, no. 1, pp. 133-155, 1986.

[13] R. Bagley and R. Calico, "Fractional order state equations for the control of viscoelastically damped structures," Journal of Guidance Control and Dynamics, vol. 14, no. 2, pp. 304-311, 1991.

[14] H. Sun, A. Abdelwahab, and B. Onaral, "Linear approximation of transfer function with a pole of fractional power," IEEE Transactions on Automatic Control, vol. 29, no. 5, pp. 441-444, 1984.

[15] C. Li and F. Zhang, "A survey on the stability of fractional dierential equations," The European Physical Journal-Special Topics, vol. 193, no. 1, pp. 27-47, 2011.

[16] B. Ahmad and A. Alsaedi, "Existence and uniqueness of solutions for coupled systems of higher-order nonlinear fractional differential equations," Fixed Point Theory and Applications, vol. 2010, Article ID 364560, 17 pages, 2010.

[17] J.-G. Lu and G. Chen, "Robust stability and stabilization of fractional-order interval systems: an LMI approach," IEEE Transactions on Automatic Control, vol. 54, no. 6, pp. 1294-1299, 2009. 
[18] J.-G. Lu and Y.-Q. Chen, "Robust stability and stabilization of fractional-order interval systems with the fractional order $0<\alpha<1$ case," IEEE Transactions on Automatic Control, vol. 55, no. 1, pp. 152-158, 2010.

[19] Y.-H. Lan and Y. Zhou, "LMI-based robust control of fractional-order uncertain linear systems," Computers \& Mathematics with Applications, vol. 62, no. 3, pp. 1460-1471, 2011.

[20] Y.-H. Lan, H.-X. Huang, and Y. Zhou, "Observer-based robust control of $a(1 \leq a x 3 c ; 2)$ fractionalorder uncertain systems: a linear matrix inequality approach," IET Control Theory $\mathcal{E}$ Applications, vol. 6, no. 2, pp. 229-234, 2012.

[21] Y. Cao and W. Ren, "Distributed formation control for fractional-order systems: dynamic interaction and absolute/relative damping," Systems \& Control Letters, vol. 59, no. 3-4, pp. 233-240, 2010.

[22] Y. Tang, Z. Wang, and J.-a. Fang, "Pinning control of fractional-order weighted complex networks," Chaos. An Interdisciplinary Journal of Nonlinear Science, vol. 19, no. 1, pp. 131121-131129, 2009.

[23] Y. Tang and J. Fang, "Synchronization of N-coupled fractional-order chaotic systems with ring connection," Communications in Nonlinear Science and Numerical Simulation, vol. 15, no. 2, pp. 401-412, 2010.

[24] W. Zhang, Y. Tang, J. Fang, and W. Zhu, “Exponential cluster synchronization of impulsive delayed genetic oscillators with external disturbances," Chaos, vol. 19, no. 1, pp. 0431371-0431379, 2011.

[25] W. Zhang, J. Fang, and Y. Tang, "Robust stability for genetic regulatory networks with linear fractional uncertainties," Communications in Nonlinear Science and Numerical Simulation, vol. 17, no. 4, pp. 1753$1765,2012$.

[26] Z. Li, Z. Duan, G. Chen, and L. Huang, "Consensus of multiagent systems and synchronization of complex networks: a unified viewpoint," IEEE Transactions on Circuits and Systems, vol. 57, no. 1, pp. 213-224, 2010.

[27] N. Laskin, "Fractional market dynamics," Physica A, vol. 287, no. 3-4, pp. 482-492, 2000.

[28] G. M. Zaslavsky and M. Edelman, "Hierarchical structures in the phase space and fractional kinetics. I. Classical systems," Chaos, vol. 10, no. 1, article 135, 12 pages, 2000.

[29] R. Hilfer, Applications of Fractional Calculus in Physics, World Scientific, 2000.

[30] I. Podlubny, Fractional Differential Equations, Academic Press, 1999.

[31] S. G. Samko, A. A. Kilbas, and O. I. Marichev, Fractional Integrals and Derivatives, Gordon and Breach Science, 1993.

[32] H. Li, "Cluster synchronization stability for stochastic complex dynamical networks with probabilistic interval time-varying delays," Journal of Physics A, vol. 44, no. 10, pp. 105101-105121, 2011.

[33] M. Moze, J. Sabatier, and A. Oustaloup, "Lmi tools for stability analysis of fractional systems," in International Design Engineering Technical Conferences and Computers and Information in Engineering Conference (IDETC/CIE '05), vol. 6, pp. 1611-1619, September 2005.

[34] P. P. Khargonekar, I. R. Petersen, and K. Zhou, "Robust stabilization of uncertain linear systems: quadratic stabilizability and $H^{\infty}$ control theory," IEEE Transactions on Automatic Control, vol. 35, no. 3, pp. 356-361, 1990. 


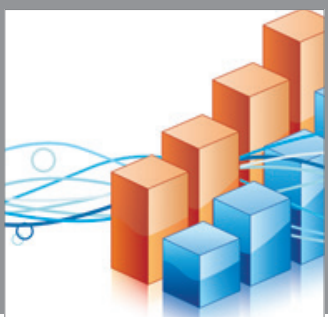

Advances in

Operations Research

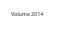

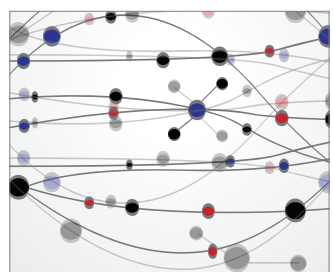

\section{The Scientific} World Journal
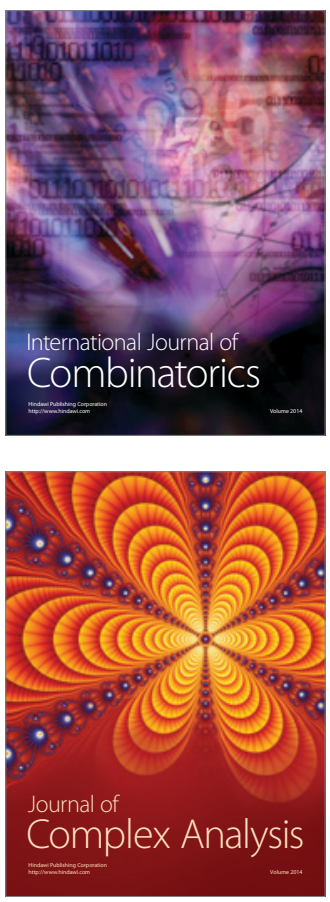

International Journal of

Mathematics and

Mathematical

Sciences
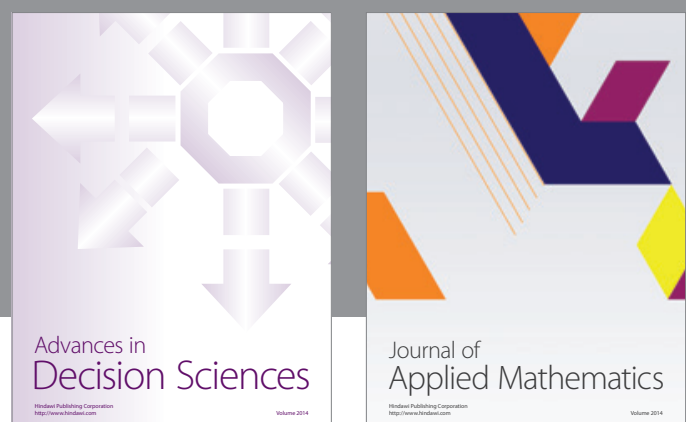

Journal of

Applied Mathematics
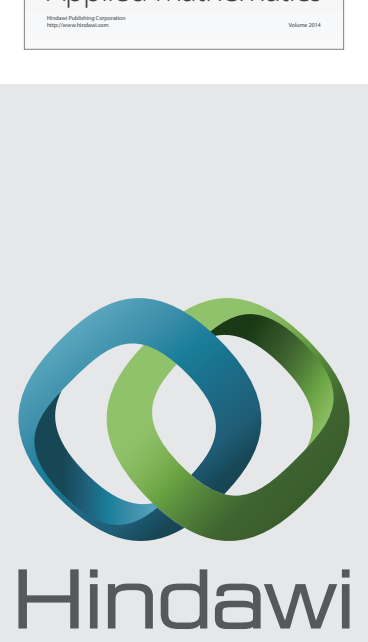

Submit your manuscripts at http://www.hindawi.com
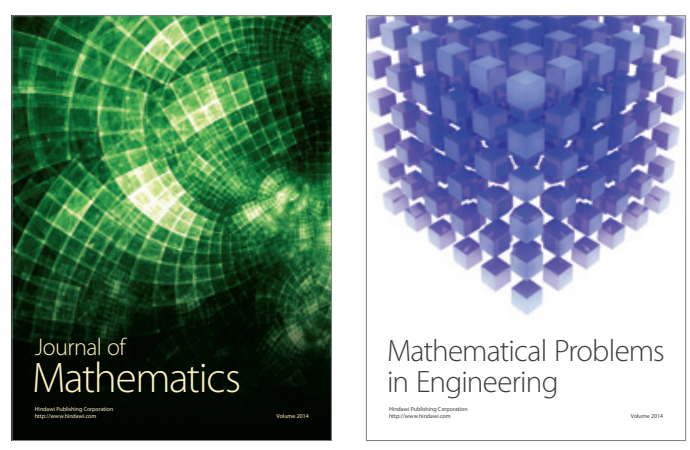

Mathematical Problems in Engineering
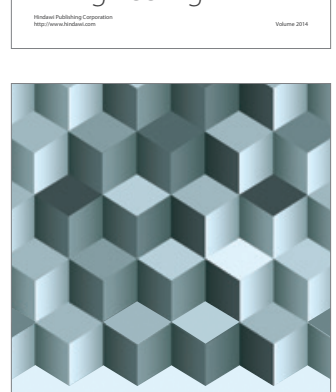

Journal of

Function Spaces
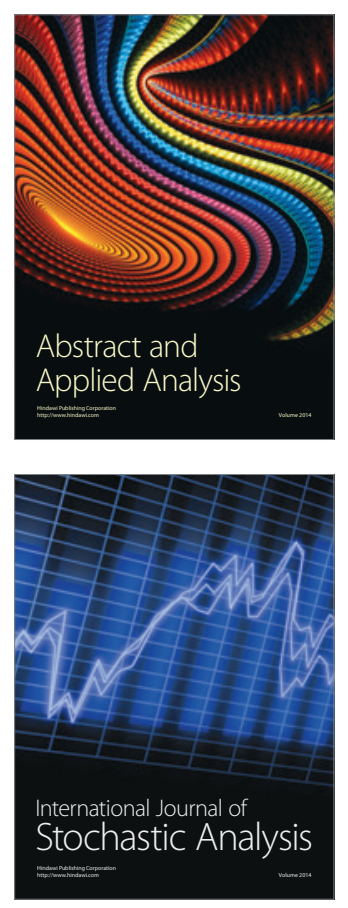

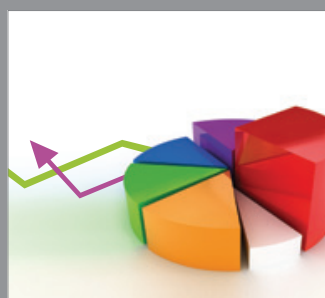

ournal of

Probability and Statistics

Promensencen
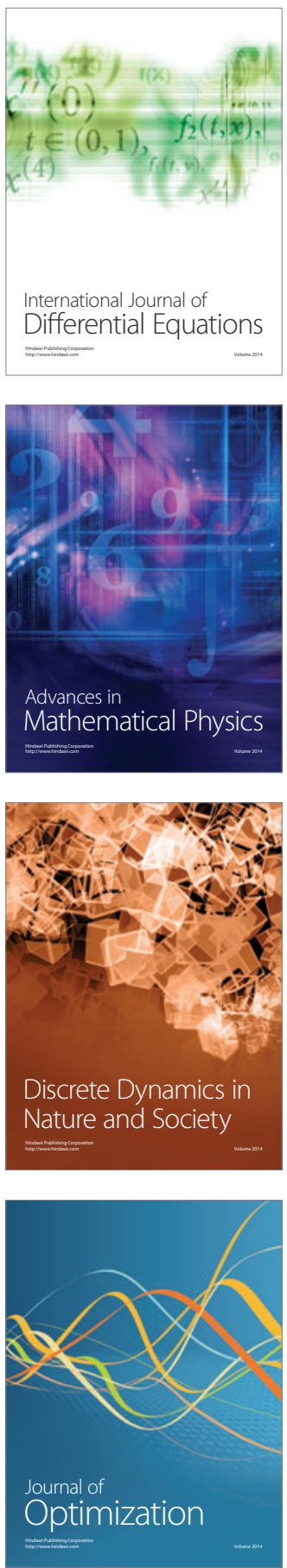